\title{
Chapter 11. Hypertension in children
}

\author{
Hypertension Research (2014) 37, 339-342; doi:10.1038/hr.2014.14
}

\section{POINT 11}

1. In children, hypertension should be evaluated with respect to age and gender. (Recommendation grade: C1, Evidence level: IVb)

2. A marked increase in blood pressure should be differentiated from secondary hypertension. (Recommendation grade: $\mathbf{C} 1$, Evidence level: IVb)

3. As essential hypertension in children can track into adult essential hypertension, lifestyle modifications should be primarily performed. (Recommendation grade: C1, Evidence level: IVb)

4. Indications for drug therapy include secondary hypertension, target organ damage, diabetes, chronic kidney disease (CKD) and so on. (Recommendation grade: C1, Evidence level: IVb)

5. For drug therapy, angiotensin-converting enzyme (ACE) inhibitors, angiotensin II receptor blockers (ARBs) and calcium (Ca) channel blockers are recommended as the first-choice drugs. (Recommendation grade: $\mathrm{C} 1$, Evidence level: IVb)

6. Even in children, blood pressure measurement should be performed during every health-care episode or through blood pressure screening. (Recommendation grade: C1, Evidence level: IVb)

\section{CHARACTERISTICS OF HYPERTENSION IN CHILDREN AND ADOLESCENTS}

On blood pressure screening, essential hypertension is detected in $\sim 1-3 \%$ of higher graders among elementary-school and high-school students. ${ }^{934,935}$ Secondary hypertension is also detected, but it is infrequent. Generally, essential hypertension is mild with no clinical symptoms, but its complication by left ventricular hypertrophy (LVH) and tracking into adult hypertension are common problems. In the School Health Safety Law, checkup items do not include blood pressure measurement, but it should be conducted in children and high-school students.

Fifty percent of children with essential hypertension are obese, and the prevalence of obesity influences that of hypertension. The number of obese children had increased gradually since 1977, but reached a plateau during the last 10 years. ${ }^{936}$

\section{BLOOD PRESSURE MEASUREMENT}

Accurate blood pressure measurement is essential for the diagnosis of hypertension. In small children, blood pressure should be measured in a seated position on the lap of a parent. The selection of an appropriate size cuff is also important. With mercury sphygmomanometers, commercially supplied cuffs of 7-cm width for 3-6-year olds, 9-cm width for 6-9-year olds and 13-cm width (adult size) for those aged 9 years or older are recommended. However, the cuff should be selected according to the size of the child's upper arm rather than their age, and one with an inflatable bladder width exceeding $40 \%$ of the arm circumference at a point midway between the olecranon and acromion and a length sufficient to cover $80 \%$ or more of the arm circumference should be used. Blood pressure should be consecutively measured three times. Considering the reproducibility, the third value should be adopted as a rule. ${ }^{937}$ However, some studies have adopted the lowest value, and many studies in the United States have adopted the mean value.

In the United States, detailed criteria for hypertension in children are determined according to gender, age and height. ${ }^{938}$ In Japan, however, there are few reports on blood pressure in children. In the Guidelines, criteria for hypertension were established based on the data obtained on blood pressure screening (Table 11-1). ${ }^{939}$ When analyzing the blood pressure levels of $\sim 40000$ elementary-school and junior-high-school students measured by the Association of Preventive Medicine, the prevalence of hypertension calculated according to the criteria is consistent with that previously reported. ${ }^{939}$ On the other hand, a study reported criteria for hypertension with respect to gender and age based on the reliable results of blood pressure measurement involving more than 10000 children in Japan. ${ }^{940}$ The criteria are $10-15 \mathrm{~mm} \mathrm{Hg}$ lower than those in the Guidelines at each age, and systolic blood pressure for first- to third-grade elementary school students is about $20 \mathrm{~mm} \mathrm{Hg}$ lower. Therefore, they are not adopted in the JSH 2014 Guidelines, because the number of subjects will increase with the use of the above criteria.

\section{PATHOLOGICAL FEATURES OF HYPERTENSION IN CHILDREN}

Hypertension detected on blood pressure screening is mostly essential hypertension. A diagnosis of essential hypertension in children is made in consideration of age (adolescence), degree of hypertension (mild), obesity, family history and lack of symptoms suggestive of secondary hypertension. Children up to the third grade may be excluded from the diagnosis of essential hypertension.

The possibility of secondary hypertension increases with a younger age or higher blood pressure. Hypertension related to renal diseases accounts for $60-80 \%$ of children with secondary hypertension, and scarred kidney (reflux nephropathy) associated with vesicoureteral reflux or chronic renal failure related to congenital renal/urinary tract abnormalities requires particular attention.

\section{OBESITY AND HYPERTENSION}

Hypertension is observed more frequently in obese children from the fourth to ninth grade $(3-5 \%)$ than in those with a standard 
Table 11-1 Criteria for hypertension in children with respect to age and gender

\begin{tabular}{lcc}
\hline & $\begin{array}{r}\text { Systolic blood pressure } \\
(\mathrm{mm} \mathrm{Hg})\end{array}$ & $\begin{array}{c}\text { Diastolic blood pressure } \\
(\mathrm{mm} \mathrm{Hg})\end{array}$ \\
\hline Pre-school children & $\geqslant 120$ & \\
Elementary school & $\geqslant 130$ & $\geqslant 80$ \\
First to third graders & $\geqslant 135$ & $\geqslant 80$ \\
Fourth to sixth graders & $\geqslant 140$ & $\geqslant 85$ \\
Junior high-school & $\geqslant 135$ & $\geqslant 80$ \\
Boys & & \\
Girls & $\geqslant 140$ & \\
High-school & & \\
\hline
\end{tabular}

body size. ${ }^{941}$ The prevalence of hypertension increases with the degree of obesity. Isolated systolic hypertension, which is a characteristic of obese children, is observed in $1.6 \%$ of boys and $3.1 \%$ of girls with mild obesity but in $8.3 \%$ of boys and $12.5 \%$ of girls with marked obesity. ${ }^{941}$ As hypertension and obesity ${ }^{942}$ in children frequently track into essential hypertension and obesity in adulthood, they should be corrected during childhood.

\section{NUTRITION OF THE FETAL PERIOD AND HYPERTENSION}

It has been shown that nutrition during embryonic and fetal development is closely involved in the occurrence of essential hypertension. According to results in Japan, blood pressure at the age of 3 years was higher with decreasing birth weight and increasing body weight. ${ }^{943}$ Moreover, in a 20-year follow-up of 4626 individuals from birth, a lower birth weight and a smaller rate of increase in height from 3 until 20 years of age were independently associated with increases in the blood pressure and serum cholesterol level at the age of 20 years, respectively. ${ }^{944}$

A study of markedly obese children reported that those with a lower birth weight were more vulnerable to metabolic syndrome including hypertension. ${ }^{945}$ According to the Vital Statistics of Japan by the Ministry of Health, Labour and Welfare, the mean birth weight (single delivery) decreased from $3.20 \mathrm{~kg}$ in 1980 to $3.02 \mathrm{~kg}$ in 2009 . In contrast, the percentage of low-birth-weight infants $(<2500 \mathrm{~g}$, single delivery) increased from $4.6 \%$ in 1980 to $8.3 \%$ in 2009 . These changes may be partly explained by the inadequate dietary intake of pregnant women, and a well-balanced diet is recommended both before and during pregnancy. Therefore, it is important to acquire an appropriate dietary habit during childhood for the prevention of lifestyle-related diseases not only in the future but also in the next generation.

\section{PROBLEMS WITH ESSENTIAL HYPERTENSION IN CHILDREN AND ADOLESCENTS}

Problems with essential hypertension in children and adolescents include complications (target organ damage) and the tracking into adult essential hypertension. As complications, LVH (increase in left ventricular muscle weight), carotid intima-media wall thickening, renal dysfunction (urinary albumin excretion) and changes in the fundic arterioles have been reported. ${ }^{937,946}$

According to the results of comparison of blood pressure at juniorhigh-school age and after 20 years in Japan, 20.9\% of hypertensive junior-high-school students were still hypertensive after 20 years, whereas $5.5 \%$ of normotensive individuals became hypertensive. ${ }^{947}$
In a study in which college students were re-examined after 8-26 years, hypertension was observed in $44.6 \%$ of the hypertensive group but in only $9.2 \%$ of the normotensive group. ${ }^{948}$ In an overseas large-scale study that followed-up 1505 children aged 5-14 years for 15 years or longer (Bogalusa Heart Study), ${ }^{949}$ twice the expected number of individuals (40\% for systolic blood pressure and 37\% for diastolic blood pressure) whose levels were in the highest quintile in childhood remained there 15 years later. The prevalence of hypertension in adulthood was much higher in individuals whose childhood blood pressure was in the top quintile: 3.6 times higher (18\% vs. $5 \%)$ in systolic blood pressure and 2.6 times higher ( $15 \%$ vs. $5.8 \%)$ in diastolic blood pressure compared with individuals in every other quintile. Therefore, nonpharmacological treatments involving therapeutic lifestyle changes and health-related behaviors are highly recommended in children and adolescents with essential hypertension in the early stage.

\section{PRIMARY PREVENTION OF HYPERTENSION IN CHILDHOOD}

It is extremely important to establish an appropriate lifestyle (dietary and exercise habits) from early childhood for the long-term prevention of lifestyle-related diseases. On checkups for the prevention of childhood lifestyle-related diseases (in school health), lifestyle, gestational age on delivery, birth weight and a family history of hypertension, including pregnancy-associated hypertension, should be inquired from children's families.

\section{1) Diet}

Treatment for obesity involves restricting energy intake, balancing nutritional intake and correcting unfavorable eating habits. Concurrent dietary and exercise therapies are more effective for the treatment of hypertension associated with obesity.

As indicated in adults, an excessive salt intake may be involved in an increase in blood pressure even in children. Salt restriction practiced from the neonatal period suppresses increases in blood pressure in childhood. ${ }^{353}$ A study involving 6235 children/adolescents (8-18 years) indicated that there was a positive correlation between salt intake and blood pressure/the risk of hypertension, and that this tendency was more marked in obese individuals. ${ }^{950}$

Salt restriction and $\mathrm{K}$ consumption should be promoted in accordance with guidelines for adults (see Chapter 4, Lifestyle modifications).

\section{2) Exercise}

For the correction of obesity, exercise for pleasure is recommended. The total amount of daily exercise is important for the prevention of increases in blood pressure regardless of the presence or absence of obesity. ${ }^{951}$

\section{MANAGEMENT OF HYPERTENSION}

Figure 11-1 shows the procedure for managing hypertension in children. In children and adolescents who are found to be mildly hypertensive through health screening, blood pressure should be measured repeatedly on different occasions. If blood pressure always exceeds the criteria, home blood pressure measurement should be performed to rule out white coat hypertension. ${ }^{952}$ Although no criteria of home blood pressure in children have been established, a value 5-mm Hg lower than clinic blood pressure should be regarded as a reference value in accordance with criteria for adults. As secondary hypertension is strongly suggested in the absence of white coat hypertension or moderate to severe obesity, close examination, primarily of the kidneys, should be performed. Twenty-four-hour 


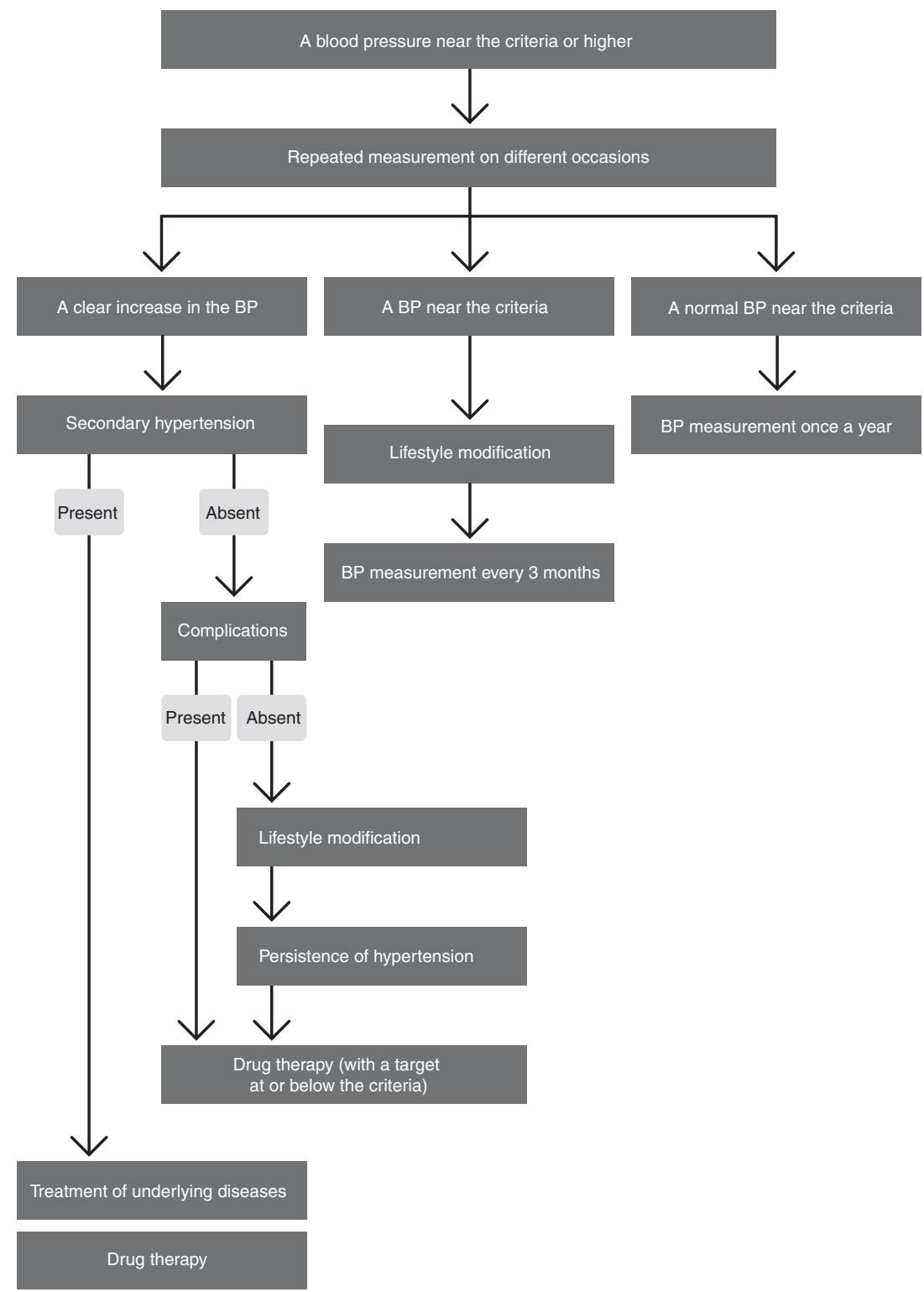

Figure 11-1 Procedure for hypertension management in children. At the same age, blood pressure is higher in taller children. Therefore, if blood pressure is approximate to the criteria, the height must also be considered. A full color version of this figure is available at the Hypertension Research journal online.

ambulatory blood pressure monitoring is useful not only for the diagnosis of white coat hypertension but also for the detection of target organ damage. ${ }^{946}$

\section{1) Nonpharmacological interventions}

As essential hypertension in children and adolescents is often mild, lifestyle modifications should be primarily performed. As there is a report of success in reducing blood pressure by restricting the salt intake of high-school students, ${ }^{935}$ salt intake should be initially reduced for dietary therapy.

In a study in which a 12-week weight control program was conducted in obese children to examine changes in blood pressure for 28 weeks, both systolic and diastolic blood pressures significantly reduced with the body weight. ${ }^{953}$ Therefore, weight control is primarily necessary in obese patients with hypertension.

Furthermore, exercise is also important in addition to caloric restriction. A study reported that moderate to intense aerobic exercise for 40 min practiced 3-5 days a week improved the vascular function, thereby reducing blood pressure ${ }^{954}$ (see Chapter 4, Lifestyle modifications).

\section{2) Drug therapy}

In hypertensive children, drug therapy is indicated for (1) secondary hypertension requiring drug therapy, (2) the concomitant development of target organ damage, (3) the presence of CKD and (4) the presence of diabetes mellitus. ${ }^{937}$

There are only a few reports on the effects of antihypertensive drugs in children. To treat LVH or CKD, ACE inhibitors or ARBs are used.

As the first-choice drugs, ACE inhibitors, ARBs and Ca channel blockers are recommended. ${ }^{937}$ A meta-analysis of 27 studies involving children showed that ACE inhibitors, ARBs and Ca channel blockers exhibited similar hypotensive effects. ${ }^{955}$ Initially, low-dose therapy with a single drug should be started, and the dose should be increased to a standard dose in $4-8$ weeks while evaluating the effects. Few 
Table 11-2 Antihypertensive drugs approved for the treatment of hypertension in children (once-a-day oral administration)

\begin{tabular}{|c|c|c|c|}
\hline & Generic name & Proprietary name (standard unit) & Dosage (daily dose) \\
\hline \multirow[t]{2}{*}{ ACE inhibitor ${ }^{a}$} & Enalapril & Renivace (2.5-, 5-, and 10-mg tablets) & Infants aged 1 month or older: $0.08 \mathrm{mg} \mathrm{kg}^{-1}$ \\
\hline & Lisinopril & Zestril, Longes, (5-, 10- and 20-mg tablets) & Children aged 6 years or older: $0.07 \mathrm{mg} \mathrm{kg}^{-1}$ (maximum dose: $20 \mathrm{mg}$ ) \\
\hline \multirow[t]{3}{*}{ ARB } & Valsartan & Diovan (20-, 40-, 80- and 160-mg tablets) & For children aged 6 years or older \\
\hline & & & Body weight of <35 kg: 20 mg (maximum dose: 40 mg) \\
\hline & & & Body weight of $\geqslant 35 \mathrm{~kg}: 40 \mathrm{mg}$ \\
\hline Ca channel blocker & Amlodipine & Norvasc and Amlodin (2.5- and 5-mg tablets/OD tablets) & Children aged 6 years or older: $2.5 \mathrm{mg}$ \\
\hline
\end{tabular}

Abbreviations: ACE, angiotensin-converting enzyme, ARB, angiotensin II receptor blockers. The doses of these drugs should be increased/decreased in accordance with age and symptoms if necessary.

${ }^{a} \mathrm{ACE}$ inhibitors are not recommended for children with renal hypofunction as a rule. If administration is required, it should be started at a low dose, and the dose should be carefully increased while checking the renal function.

studies have reported the use of diuretics in children. In 2012, an antihypertensive drug to be indicated for hypertension in children was approved for the first time in Japan (Table 11-2). The dose of an ACE inhibitor, enalapril maleate, is established for infants aged 1 month or older, but those of other antihypertensive drugs for children aged 6 years or older. However, these doses should be increased/ decreased in accordance with age, body weight and symptoms if necessary.

\section{3) Antihypertensive drugs for special situations}

In hypertensive adults with kidney disease, diabetes mellitus or cardiovascular disease, strict blood pressure control is necessary. In children with $\mathrm{CKD}$, the renal prognosis can also be improved by maintaining blood pressure below the normal range using ACE inhibitors. ${ }^{956}$ Furthermore, strict blood pressure control with ACE inhibitors reduces $\mathrm{LVH}^{957}$

\section{Citation Information}

We recommend that any citations to information in the Guidelines are presented in the following format:

The Japanese Society of Hypertension Guidelines for the Management of Hypertension (JSH 2014). Hypertens Res 2014; 37: 253-392.

Please refer to the title page for the full list of authors. 